\title{
Polymorphonuclear leukocyte isolation from venous blood of the dogs*
}

\section{Research Article}

Volume: 3, Issue: 3 December 2019

Pages: $75-78$

\author{
Güneş Karakurt, Kader Yıldız \\ Kırıkkale University, Graduate School of Health Science, Department of Parasitology \\ Turkey, Karakurt G.: ORCID: 0000-0001-7564-516X; Yıldız K.: ORCID: 0000-0001-5802-6156
}

\author{
Article History \\ Received: 17. 09.2019 \\ Accepted: 13.12 .2019 \\ Available online: \\ 13. 12.2019
}

\begin{abstract}
The current study aimed to isolate neutrophils from venous blood samples of healthy dogs. Venous blood samples were obtained from Venae cephalica of clinically healthy dogs ( $n: 5)$ into heparinized tubes. The blood samples $(2 \mathrm{~mL})$ and Percoll dilutions $(45 \%, 54 \%, 63 \%$, and 72\%) prepared with Hanks Balanced Salt Solution were layered into sterile tubes. After centrifuge, the polymorphonuclear leukocytes (PMN) were aspirated between $63 \%$ and $72 \%$ interfaces of the Percoll dilutions into tubes. The samples of PMN observed under a light microscope. Viability was detected microscopically after stained with trypan blue dye. DiffQuick staining was used to detect neutrophil purity of the isolated PMN. In the present study, the neutrophils ratio was calculated as $92 \%$ of the isolated polymorphonuclear cells. The neutrophil viability was calculated as $98 \%$ of PMNs isolated from the venous blood samples of healthy dogs. In the present study, the Percoll gradient centrifugation $(72 \%, 63 \%, 54 \%$, and $45 \%$ ) is a fast technic for isolation of the neutrophils from venous blood samples of dogs.
\end{abstract}

Keywords: dog; venous blood; polymorphonuclear leukocyte; neutrophil; isolation

DOI: $10.30704 / \mathrm{http}-w w w-j i v s-n e t .621263$

To cite this article: Karakurt, G., Yıdız K. (2019). Polymorphonuclear leukocyte isolation from venous blood of the dogs. Journal of Istanbul Veterinary Sciences. 3(3), 75-78, Abbreviated Title: J Ist Vet Sci

*The part of this paper was presented at the International VETEXPO Veterinary Science Congress in September 20-22, 2019, İstanbul/Turkey. *This study is summarized from the first author's PhD Thesis

\section{Introduction}

Polymorphonuclear leukocyte (PMN), granular leukocyte or granulocyte, is a type of white blood cell. The PMN has been commonly recognized histologically by their cytoplasmic appearance and nuclear shapes. The PMN consist of some granulocytic cells called neutrophils, eosinophils, and basophils. Most of the PMN are neutrophils, which ratio varies depending on the animal species. (Kaplan and Radic, 2012). Mammalian neutrophils possess multi-lobed nuclei (usually two and five lobes). The neutrophils produce from bone marrow (about 7 million per minute) and control by the homeostatic balance in the organism. The number of circulating neutrophils increases in some situations such as stress and infection (Rosales, 2018). The neutrophils can live 3-12 hours in the organism, which can be as long as 1-2 days (Kruger et al., 2015). Cytokines and bacterial products prolong the life span of neutrophils. If it does not enter the process of inflammation, neutrophils are phagocytosed by macrophages in the organism (Mayadas et al., 2014). The innate immune system is a first defence mechanism that provides rapid protection after confronted with infectious microorganisms in the body (Kaplan and Radic, 2012). Neutrophils are one of the most important components of innate immunity. Following the pathogenic invasion, neutrophils respond

*Corresponding Author: Güneş Karakurt

https://dergipark.org.tr/tr/pub/http-www-jivs-net 
to chemotactic stimuli and migrate to the infection area and use a variety of defence strategies including phagocytosis, degranulation and netosis to fight pathogens (Selders et al., 2017).

Canine neutrophils have been studied for different in vitro studies (Bosco et al. 2013; Sheats et al. 2015; Li et al. 2018). The purpose of this study was to isolate neutrophils from venous blood samples of healthy dogs. Although there are many neutrophil isolation methods, these methods do not apply to all animals (Oh et al., 2008; Marchi et al., 2013; Yildiz et al., 2017). In the present study, it was aimed to isolation of neutrophils from venous blood samples of healthy dogs by using discontinuous Percoll dilutions.

\section{Material and methods}

All experimental procedures have been approved by the Ethics Commission of Kırıkkale University (06.09.2017, no:17/32). Venous blood samples (5 mL) were obtained from Venae cephalica of clinically healthy dogs $(n: 5)$ into heparinized tubes. The methodology was a modified version of Sursal et al. (2018). Briefly, Percoll dilutions (45\%, 54\%, 63\%, and 72\%) were prepared with Hanks Balanced Salt Solution (Sigma). Then, two $\mathrm{mL}$ of each Percoll dilution starting from higher concentration carefully layered into a polystyrene centrifuge tube. The blood samples $(2 \mathrm{~mL})$ were layered on the lowest concentration of Percoll dilution $(45 \%)$ in the tubes after diluted with PBS-EDTA (0.02\%) (1:1). The tubes centrifuged at room temperature $(500 \times \mathrm{g}, 35 \mathrm{~min})$ (Thermo Scientific, SL 16R). Three cell layers were observed among the Percoll dilutions after the completed centrifuge step. The cell layer was aspirated between $63 \%$ and $72 \%$ interfaces into sterile tubes. The tubes centrifuged at $+4^{\circ} \mathrm{C}$ ( $300 \times \mathrm{g}, 10$ minutes) and the supernatant was removed, then the PMN was diluted in RPMI-1640 (without phenol red, Sigma). To detect PMN numbers, the samples of PMN put on the Neubauer chamber and observed under a light microscope (Leica DM750).

The PMN samples were smeared on the slides. After dried on air and fixation with methyl alcohol, Diff -Quick staining (Bio Optica, Italy) was used to detect neutrophil purity of the isolated PMN. All polymorphonuclear cells were counted in ten microscopic areas randomly selected. Then, the ratio of neutrophils was compared to all polymorphonuclear cells counted. Trypan blue dye was used to detect the viability of isolated neutrophils. The PMN samples were stained with trypan blue dye (1:1) in the sterile microtubes. The cells stained with trypan blue dye were evaluated as died under the light microscope. To detect the neutrophil viability, all polymorphonuclear cells (stained and unstained) were counted in ten microscopic areas randomly selected. Then, the ratio of cells stained with trypan blue was compared to all polymorphonuclear cells counted. The PMN isolations were repeated three times.

\section{Results}

PMNs were detected between $63 \%$ and $72 \%$ interfaces of the Percoll dilutions after centrifuging the tubes. Figure 1 shows the PMNs of dogs in Neubauer chamber. After Diff-Quick staining, the neutrophils were easily observed as a dark blue lobulated nucleus and pale pink cytoplasm in the slides under the light microscope (Figure 2). The neutrophils ratio was calculated as $92 \%$ of the isolated polymorphonuclear cells. The neutrophil viability was calculated as $98 \%$ of PMNs isolated from the venous blood samples of healthy dogs.

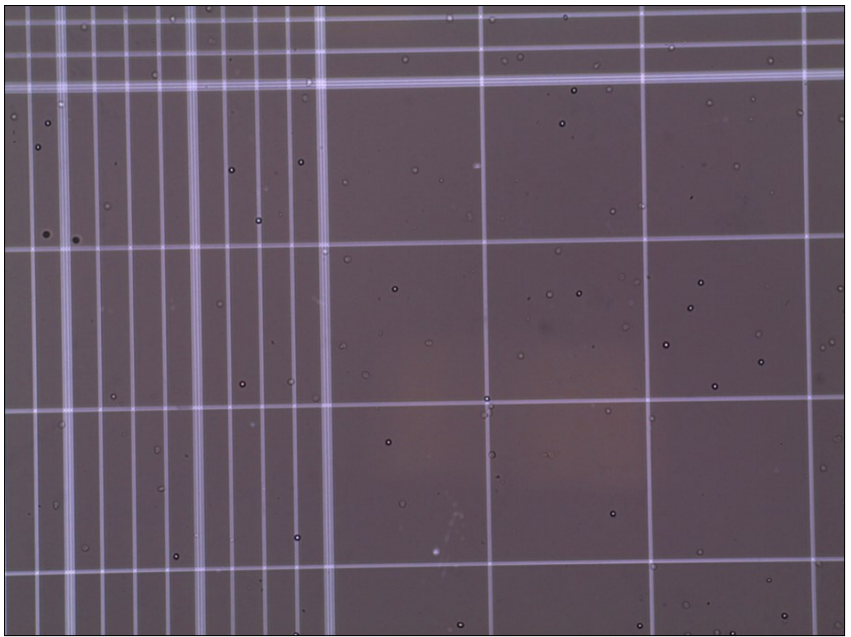

Figure 1. Light microscopic observation of the polymorphonuclear leucocytes of the dogs in the Neubauer chamber. X20.

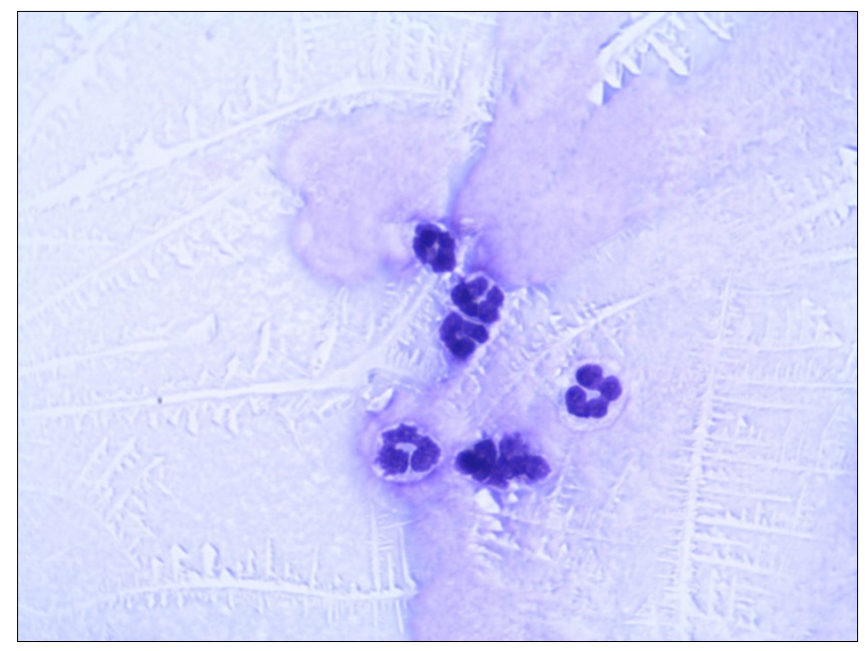

Figure 2. The light microscopic view of the isolated neutrophils with a dark blue lobulated nucleus and pale pink cytoplasm. Diff-Quick staining, x100. 


\section{Discussion}

Polymorphonuclear leucocyte population consists mainly of neutrophils (Kaplan and Radic, 2012; Patel and Chatterjee, 2018). Neutrophils play an important role in maintaining innate host defence against pathogens in dogs. In general, in vitro experiments design to understand neutrophil functions during the defence to infectious microorganisms in the organism (Gosset et al., 1983; Yildiz et al., 2017; Lawson et al., 2018; Yildiz et al., 2019). Neutrophil separation can be accomplished by centrifugation with different density gradient solutions. Some of them include Percoll, Biocoll, Ficoll-Hypaque, and Sucrose Polymerdiatrizoate gradient are employed for the cell separation. There are some protocols are used for neutrophils isolation from dogs venous blood samples (Sano et al. 2004; Jeffery et al. 2016; Wei et al. 2016). Some of them are dextran sedimentation and FicollHypaque (Sano et al. 2004; Jeffery et al. 2016). Some authors prefer a commercially available canine PMN isolation kit to obtain PMN from blood samples of dogs (Wei et al.2016). Percoll, colloidal silica particles coated with polyvinylpyrrolidone, is a gradient medium for separation of cells, subcellular particles and even viruses. Different Percoll dilutions are used

\section{References}

Bosco, A. M., de Almeida, B. F. M., Pereira, P. P., Narciso, L. G., Lima, V. M. F. \& Ciarlini, P.C. (2013). High concentrations of glucose reduce the oxidative metabolism of dog neutrophils in vitro. BMC Veterinary Research, 9, 24.

Cools-Lartigue, J., Spicer, J., McDonald, B., Gowing, S., Chow, S., Giannias, B., Bourdeau, F., Kubes, P. \& Ferri, L. (2013). Neutrophil extracellular traps sequester circulating tumor cells and promote metastasis. Journal of Clinical Investigation, 123, 3446-3458.

Gosset, K.A.,MacWilliams P.S., Enright F.M. \& Cleghorn B. (1983). In vitro function of canine neutrophils during experimental inflammatory disease. Veterinary Immunology and Immunopathology, 5, 151-159.

Kaplan, M.J , Radic, M. (2012). Neutrophil extracellular traps: double-edged swords of innate immunity. Journal of Immunology, 189, 2689-2695.

Kruger $\mathrm{P}$, Saffarzadeh M, Weber AN, Rieber N, Radsak M, Bernuth H, Benarafa C, Roos D, Skokowa J, Hartl D (2015). Neutrophils: between host defence, immune modulation, and tissue injury. PLoS Pathogens, 11, e1004651. for isolation of PMN from venous blood samples of different animals and human (Mosca and Forte, 2016; Cools-Lartigue et al. 2013; Swamydas et al. 2015; Sursal et al. 2018). Feline neutrophils are successfully obtained using the Percoll dilutions ( $72 \%, 63 \%, 54 \%$, and $45 \%$ ) (Sursal et al. 2018). In the present study, the Percoll gradient centrifugation $(72 \%, 63 \%, 54 \%$, and $45 \%$ ) was useful for isolation of the neutrophils from venous blood samples of dogs. This technic was fast and simple rather than the other PMN isolation technics.

In conclusion, Percoll gradient centrifugation was used to obtain neutrophils from venous blood samples of healthy dogs. This method is useful for isolation dog neutrophils.

\section{Acknowledgement}

This study was financially supported by Kırıkkale University Scientific Research Coordination Unit (Project number: 2017/080).

Lawson, C., Smith, S.A., O'Brien, M.\& McMichael, M. (2018). Neutrophil extracellular traps in plasma from dogs with immune-mediated haemolytic anemia. Journal of Veterinary Internal Medicine 32, 128-134.

Jeffery U, Gray R. D., \& LeVine D. N. (2016). A Simple Fluorescence Assay for Quantification of Canine Neutrophil Extracellular Trap Release. Journal of Visualized Experiments, 21(117),1-4.

Li, R. H. L. \& Tablin, F. (2018). In vitro canine neutrophil extracellular trap formation: dynamic and quantitative analysis by fluorescence microscopy. Journal of Visualised Experiments, 138,

Li, R. H. L., Johnson, L. R., Kohen, C. \& Tablin, F. (2018). A novel approach to identifying and quantifying neutrophil extracellular trap formation in septic dogs using immunofluorescence microscopy. BMC Veterinary Research, 14, 210.

Marchi, L. F., Sesti-Costa, R., Chedraoui-Silva, S.\& Mantovani, B. (2013). Neutrophil isolation protocol. Comparative Clinical Pathology 23(5),1469-1476

Mayadas, T. N., Cullere,X. \& Lowell, C. A. (2014). The multifaceted functions of neutrophils. Annual Review of Pathology, 9: 181-218. 
Mosca, T. \& Forte W.C. (2016). Comparative efficiency and impact on the activity of blood neutrophils isolated by Percoll, Ficoll and spontaneous sedimentation methods. Immunological investigations, 45, 29-37.

Oh, H., Siano, B.\& Diamond, S. (2008). Neutrophil Isolation Protocol. Journal of Visualized Experiments, (17), e745.

Patel, P. \& Chatterjee, S. (2018). Innate and adaptive immunity: Barriers and receptor-based recognition, immunity and inflammation in health and disease emerging roles of nutraceuticals and functional Foods in Immune Support , 3-13

Rosales, C. (2018). Neutrophil: A cell with many roles in inflammation or several cell types? Frontiers Physiology, 9, 113.

Sano J, Oguma K, Kano R, Tsumagari S, Hasegawa A. (2004), Decreased apoptotic polymorphonuclear leukocyte rate in dogs with pyometra., Journal of Veterinary Medical Science, 66(1):103-5

Selders, G. S., Fetz, A.E.,Radic, M.Z. \& Bowlin, G.L. Y (2017).An overview of the role of neutrophils in innate immunity, inflammation and host-biomaterial integration. Regenerative Biomaterials, 4, 55-68.

Sheats, M.K., Sung, E.J., Adler, K.B.\& Jones, S.L. (2015). In vitro neutrophil migration requires protein kinase c-delta ( $\delta-P K C)$ mediated MARCKS (Myristoylated
Alanine Rich C-Kinase Substrate) phosphorylation. Inflammation, 38, 1126-1141.

Sursal, N., Cakmak, A. \& Yildiz, K. (2018). Neutrophil isolation from feline blood using discontinuous Percoll dilutions. Tierarztliche Praxis Ausgabe K: Kleintiere - Heimtiere, 46, 399-402.

Swamydas, M., Luo, Y., Dorf,M.E. \& Lionakis, M.S. (2015). Isolation of mouse neutrophils. Current Protocol of Immunology, 110, 3.20.1-3.20.15.

Wei Z, Hermosilla C, Taubert A, He X, Wang X, Gong P, Li J, Yang Z, Zhang X (2004) Canine neutrophil extracellular traps release induced by the apicomplexan parasite neospora caninum in vitro, front. Immunol., 31 October 2016 https:// doi.org/10.3389/ fimmu.2016.00436

Yildiz, K., Gokpinar, S., Gazyagci, A. N., Babur, C., Sursal, N. \& Azkur, A.K. (2017). Role of NETs in the difference in host susceptibility to Toxoplasma gondii between sheep and cattle. Veterinary Immunology and Immunopathology, 189, 1-10.

Yildiz, K., Gokpinar, S., Sursal, N., Babur, C., Ozen, D. \& Azkur, A.K. (2019). Extracellular trap formation by donkey polymorphonuclear neutrophils against Toxoplasma gondii. Journal of Equine Veterinary Science, 73, 1-9. 